\title{
CLASSICAL PRETZEL KNOTS AND LEFT ORDERABILITY
}

\author{
ARAFAT KHAN AND ANH T. TRAN
}

\begin{abstract}
We consider the classical pretzel knots $P\left(a_{1}, a_{2}, a_{3}\right)$, where $a_{1}, a_{2}, a_{3}$ are positive odd integers. By using continuous paths of elliptic $\mathrm{SL}_{2}(\mathbb{R})$-representations, we show that (i) the 3 -manifold obtained by $\frac{m}{l}$-surgery on $P\left(a_{1}, a_{2}, a_{3}\right)$ has left orderable fundamental group if $\frac{m}{l}<1$, and (ii) the $n^{\text {th }}$-cyclic branched cover of $P\left(a_{1}, a_{2}, a_{3}\right)$ has left orderable fundamental group if $n>2 \pi / \arccos \left(1-2 /\left(1+a_{1} a_{2}+a_{2} a_{3}+a_{3} a_{1}\right)\right)$.
\end{abstract}

\section{INTRODUCTION}

A non-trivial group $G$ is called left orderable if it admits a total ordering $<$ such that $g<h$ implies $f g<f h$ for all elements $f, g, h$ in $G$. We study the left orderability of the fundamental groups of 3-manifolds. This study is motivated by the L-space conjecture of Boyer-Gordon-Watson [BGW] which states that an irreducible rational homology 3sphere is an L-space if and only if its fundamental group is not left orderable. Here a rational homology 3-sphere $Y$ is an L-space if its Heegaard Floer homology $\widehat{\mathrm{HF}}(Y)$ has rank equal to the order of $H_{1}(Y ; \mathbb{Z})[\mathrm{OS}]$. We will focus on 3-manifolds obtained by Dehn surgeries along a knot in $S^{3}$ or by taking cyclic branched covers of a knot.

For a rational knot $C(k, l)$ in the Conway notation or the $(-2,3,7)$-pretzel knot, some intervals of slopes for which the 3-manifold obtained from $S^{3}$ by Dehn surgery along the knot has left orderable fundamental group was determined in [BGW, HT1, HT2, $\operatorname{Tr} 1, \operatorname{Tr} 4, \mathrm{Ga} 2$, KTT, Va] by using continuous paths of elliptic and hyperbolic $\mathrm{SL}_{2}(\mathbb{R})$ representations of the knot group and the fact that the universal covering group of $\mathrm{SL}_{2}(\mathbb{R})$ is a left orderable group. In the case of the figure eight knot, by using taut foliations it was proved in $[\mathrm{Zu}]$ that any non-trivial surgery on the $n^{\text {th }}$-cyclic branched cover of the figure-eight knot has left orderable fundamental group, see also [Hu2].

A sufficient condition for the fundamental group of the $n^{\text {th }}$-cyclic branched cover of $S^{3}$ along a prime knot to be left orderable was given in [BGW, Hul] in terms of $\mathrm{SL}_{2}(\mathbb{R})$ representations of the knot group. As an application, it was proved in [Go] that for any rational knot $K$ with non-zero signature the fundamental group of the $n^{\text {th }}$-cyclic branched cover of $S^{3}$ along $K$ is left orderable for sufficiently large $n$, see also [Hu1, Tr3]. For a rational knot $C(k, l)$ or $C(2 n+1,2,2)$ in the Conway notation, the left orderability of the fundamental groups of the cyclic branched covers of $S^{3}$ along the knot was also determined in $[\mathrm{DPT}, \mathrm{Tr} 2, \mathrm{Tu}]$.

A systematic approach to the understanding of the left orderability of the fundamental groups of 3-manifolds obtained by Dehn surgeries along a knot in $S^{3}$ or by taking cyclic branched covers of a knot was proposed by Culler-Dunfield [CD], by using continuous paths of elliptic $\mathrm{SL}_{2}(\mathbb{R})$-representations of the knot group. A similar approach using

2010 Mathematics Subject Classification. 57M27, 57M25.

Key words and phrases. cyclic branched cover, Dehn surgery, L-space, left orderable group, pretzel knot, representation. 
hyperbolic $\mathrm{SL}_{2}(\mathbb{R})$-representations was also proposed by Gao [Ga1]. A geometric approach using taut foliations was proposed in $[\mathrm{Zu}]$ and references therein.

In this paper, we consider the classical pretzel knots $P\left(a_{1}, a_{2}, a_{3}\right)$, where $a_{1}, a_{2}, a_{3}$ are positive odd integers. By using continuous paths of elliptic $\mathrm{SL}_{2}(\mathbb{R})$-representations, we will prove the following.

Theorem 1. Let $a_{1}, a_{2}, a_{3}$ be positive odd integers. Then

(i) the 3-manifold obtained by $\frac{m}{l}$-surgery on $P\left(a_{1}, a_{2}, a_{3}\right)$ has left orderable fundamental group if $\frac{m}{l}<1$, and

(ii) the $n^{\text {th }}$-cyclic branched cover of $P\left(a_{1}, a_{2}, a_{3}\right)$ has left orderable fundamental group if $n>2 \pi / \arccos \left(1-2 /\left(1+a_{1} a_{2}+a_{2} a_{3}+a_{3} a_{1}\right)\right)$.

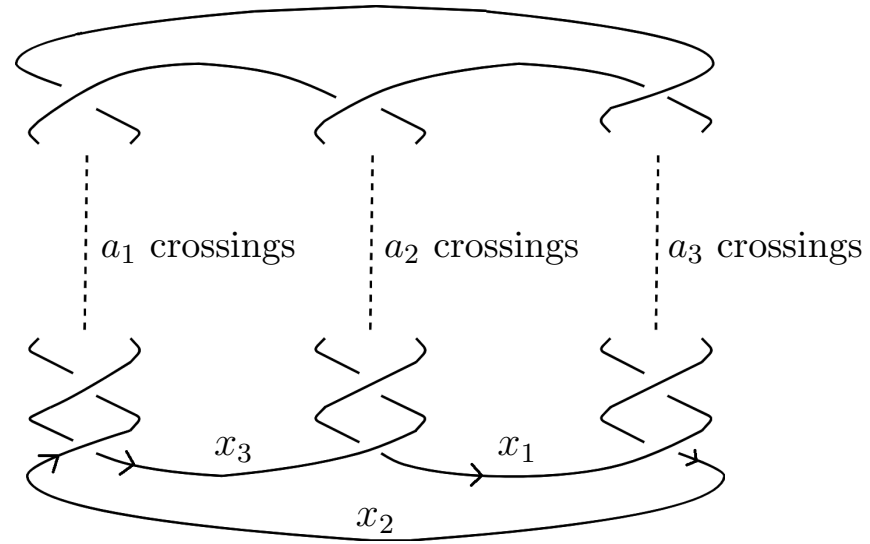

Figure 1. The pretzel knot $P\left(a_{1}, a_{2}, a_{3}\right)$.

The pretzel knot $P\left(a_{1}, a_{2}, a_{3}\right)$, where $a_{1}, a_{2}, a_{3}$ are positive odd integers, is a non-torus alternating knot and hence any non-trivial surgery along it yields a 3-manifold which is not an L-space, see [OS]. Moreover, Boileau-Boyer-Gordon [BBG] proved that the $n^{\text {th }}$ cyclic branched cover of $P\left(a_{1}, a_{2}, a_{3}\right)$ is not an L-space if $n>2 \pi / \arccos \left(1-2 /\left(1+a_{1} a_{2}+\right.\right.$ $\left.\left.a_{2} a_{3}+a_{3} a_{1}\right)\right)$. So our results support the L-space conjecture.

The paper is organized as follows. In Section 2 we recall a description of $\mathrm{SL}_{2}(\mathbb{C})$ representations of odd classical pretzel knots and then we study $\mathrm{SL}_{2}(\mathbb{R})$-representations of these knots. In Section 3 we use continuous families of $\mathrm{SL}_{2}(\mathbb{R})$-representations constructed in Section 2 to give a proof of Theorem 1.

\section{2. $\mathrm{SL}_{2}(\mathbb{R})$-REPRESENTATIONS OF PRETZEL KNOTS}

In this section we study $\mathrm{SL}_{2}(\mathbb{R})$-representations of pretzel knots $P\left(a_{1}, a_{2}, a_{3}\right)$ where $a_{1}, a_{2}, a_{3}$ are positive odd integers. We first recall the Chebyshev polynomials of the second kind and prove their properties. Then we recall a description of $\mathrm{SL}_{2}(\mathbb{C})$-representations of $P\left(a_{1}, a_{2}, a_{3}\right)$ in [Ha]. Finally, we prove the existence and continuity of a family of $\mathrm{SL}_{2}(\mathbb{R})$-representations. We also prove properties of this family.

2.1. Chebychev polynomials. Let $\left\{S_{j}(z)\right\}_{j \in \mathbb{Z}}$ be the sequence of Chebychev polynomials in the variable $z$ defined by $S_{0}(z)=1, S_{1}(z)=z$ and $S_{j}(z)=z S_{j-1}(z)-S_{j-2}(z)$ for all integers $j$. By induction we have $S_{j}( \pm 2)=( \pm 1)^{j}(j+1)$ and $S_{j}(z)=\frac{s^{j+1}-s^{-(j+1)}}{s-s^{-1}}$ for $z=s+s^{-1} \neq \pm 2$. Using this fact, one can prove the following, see e.g. [KTT]. 
Lemma 2.1. We have $S_{n}^{2}(z)-S_{n+1}(z) S_{n-1}(z)=S_{n}^{2}(z)+S_{n-1}^{2}(z)-z S_{n}(z) S_{n-1}(z)=1$.

Lemma 2.2. Fix $z \geq 2$. Then $\frac{S_{n}(z)}{S_{n-1}(z)}$ is a strictly decreasing function in $n \in \mathbb{N}$.

Proof. By Lemma 2.1 we have $S_{n}^{2}(z)-S_{n+1}(z) S_{n-1}(z)=1$. Hence $\frac{S_{n}(z)}{S_{n-1}(z)}-\frac{S_{n+1}(z)}{S_{n}(z)}=$ $\frac{1}{S_{n}(z) S_{n-1}(z)}>0$ for $z \geq 2$. The lemma follows.

Lemma 2.3. Fix $n \in \mathbb{N}$. Let $p(z)=\frac{S_{n}(z)}{S_{n-1}(z)}$ for $z \in(2, \infty)$. Then both $p$ and $\frac{z-2}{p-1}$ are both strictly increasing functions in $z$.

Proof. Write $z=s+s^{-1}$ where $s=\frac{1}{2}\left(z+\sqrt{z^{2}-4}\right)>1$. Then $p(z)=\frac{S_{n}(z)}{S_{n-1}(z)}=\frac{s^{n+1}-s^{n-1}}{s^{n}-s^{-n}}$.

Note that $\frac{d p}{d z}=\frac{d p}{d s} / \frac{d z}{d s}=\frac{d p}{d s} /\left(1-s^{-2}\right)$. To prove $p(z)$ is stricly increasing in $(2, \infty)$, it suffices to show $g(s):=\frac{s^{n+1}-s^{-n-1}}{s^{n}-s^{-n}}$ is a strictly increasing function in $(1, \infty)$. We have

$$
\begin{aligned}
s\left(s^{n}-s^{-n}\right)^{2} g^{\prime}(s) & =(n+1)\left(s^{n+1}+s^{-n-1}\right)\left(s^{n}-s^{-n}\right)-n\left(s^{n+1}-s^{-n-1}\right)\left(s^{n}+s^{-n}\right) \\
& =\left(s^{n+1}+s^{-n-1}\right)\left(s^{n}-s^{-n}\right)-2 n\left(s-s^{-1}\right) \\
& >2\left(s^{n}-s^{-n}\right)-2 n\left(s-s^{-1}\right)>0 .
\end{aligned}
$$

Here we used $\frac{s^{n}-s^{-n}}{s-s^{-1}}=S_{n-1}(z)>S_{n-1}(2)=n$ in the last inequality. Hence $g^{\prime}(s)>0$.

By a direct calculation we have

$$
\frac{z-2}{p-1}=\frac{s^{-1}(s-1)^{2}}{(s-1)\left(s^{n}+s^{-n-1}\right) /\left(s^{n}-s^{-n}\right)}=\frac{(s-1)\left(s^{2 n}-1\right)}{s^{2 n+1}+1}=1-\frac{s^{2 n}+s}{s^{2 n+1}+1} .
$$

To prove $\frac{z-2}{p-1}$ is stricly increasing in $(2, \infty)$, it suffices to show that $h(s):=-\frac{s^{2 n}+s}{s^{2 n+1}+1}$ is a strictly increasing function in $(1, \infty)$. We have

$$
\begin{aligned}
\left(s^{2 n+1}+1\right)^{2} h^{\prime}(s) & =-\left(2 n s^{2 n-1}+1\right)\left(s^{2 n+1}+1\right)+(2 n+1)\left(s^{2 n}+s\right) s^{2 n} \\
& =\left(s^{4 n}-1\right)+2 n\left(s^{2}-1\right) s^{2 n-1}>0 .
\end{aligned}
$$

Hence $h^{\prime}(s)>0$ and the lemma follows.

2.2. $\mathbf{S L}_{2}(\mathbb{C})$-representations. For a knot $K$ in $S^{3}$, let $G(K)$ denote the knot group of $K$ which is the fundamental group of the complement of an open tubular neighborhood of $K$.

It is known that a pretzel link $P\left(a_{1}, a_{2}, \cdots, a_{l}\right)$ is isotopic to any pretzel link obtained by a cyclic permutation or reversing the order of $a_{1}, a_{2}, \cdots, a_{l}$. In the case $l=3$, this implies that $P\left(a_{1}, a_{2}, a_{3}\right)$ is isotopic to $P\left(a_{f(1)}, a_{f(2)}, a_{f(3)}\right)$ for any bijection $f:\{1,2,3\} \rightarrow$ $\{1,2,3\}$.

From now on we consider the pretzel knot $P\left(a_{1}, a_{2}, a_{3}\right)$ where $a_{1}, a_{2}, a_{3}$ are positive odd integers. By the above argument we can always assume that $a_{1} \leq a_{2} \leq a_{3}$.

Write $a_{j}=2 k_{j}+1$ for $j=1,2,3$. An application of the Wirtinger algorithm to the knot diagram of $K:=P\left(a_{1}, a_{2}, a_{3}\right)$ in Figure 1 shows that the knot group $G(K)$ has a presentation with 3 generators $x_{1}, x_{2}, x_{3}$ and 3 relations

$$
\begin{aligned}
& \left(x_{2} x_{3}^{-1}\right)^{k_{1}+1} x_{3}\left(x_{2} x_{3}^{-1}\right)^{-k_{1}-1}=\left(x_{1} x_{2}^{-1}\right)^{k_{3}} x_{1}\left(x_{1} x_{2}^{-1}\right)^{-k_{3}}, \\
& \left(x_{3} x_{1}^{-1}\right)^{k_{2}+1} x_{1}\left(x_{3} x_{1}^{-1}\right)^{-k_{2}-1}=\left(x_{2} x_{3}^{-1}\right)^{k_{1}} x_{2}\left(x_{2} x_{3}^{-1}\right)^{-k_{1}}, \\
& \left(x_{1} x_{2}^{-1}\right)^{k_{3}+1} x_{2}\left(x_{1} x_{2}^{-1}\right)^{-k_{3}-1}=\left(x_{3} x_{1}^{-1}\right)^{k_{2}} x_{3}\left(x_{3} x_{1}^{-1}\right)^{-k_{2}},
\end{aligned}
$$

where $x_{1}, x_{2}, x_{3}$ are the meridians depicted in Figure 1. Note that we actually just need 2 of the above 3 relations. 
For a representation $\rho:\left\langle x_{1}, x_{2}, x_{3}\right\rangle \rightarrow \mathrm{SL}_{2}(\mathbb{C})$ we let $t_{j}:=\operatorname{tr} \rho\left(x_{j}\right), r_{1}:=\operatorname{tr} \rho\left(x_{2} x_{3}^{-1}\right)$, $r_{2}:=\operatorname{tr} \rho\left(x_{3} x_{1}^{-1}\right), r_{3}:=\operatorname{tr} \rho\left(x_{1} x_{2}^{-1}\right)$ and $r:=\operatorname{tr} \rho\left(x_{1} x_{2} x_{3}\right)$. Let

$$
\sigma_{1}:=r_{1}+r_{2}+r_{3}, \quad \sigma_{2}:=r_{1} r_{2}+r_{2} r_{3}+r_{3} r_{1}, \quad \sigma_{3}:=r_{1} r_{2} r_{3}
$$

Note that if $\rho:\left\langle x_{1}, x_{2}, x_{3}\right\rangle \rightarrow \mathrm{SL}_{2}(\mathbb{C})$ extends to a representation $\rho: G(K) \rightarrow \mathrm{SL}_{2}(\mathbb{C})$ then we must have $t_{1}=t_{2}=t_{3}$, since $x_{1}, x_{2}, x_{3}$ are conjugate to each other in $G(K)$.

By [Ha, Theorem 3.12] a representation $\rho:\left\langle x_{1}, x_{2}, x_{3}\right\rangle \rightarrow \mathrm{SL}_{2}(\mathbb{C})$, with $t_{1}=t_{2}=t_{3} \neq 0$ and $\sigma_{1}+2-2 \gamma \neq 0$, extends to an irreducible representation $\rho: G(K) \rightarrow \mathrm{SL}_{2}(\mathbb{C})$ if the following equations are satisfied

$$
\begin{aligned}
\left(\gamma-2-r_{j}\right) S_{k_{j}}\left(r_{j}\right) & =\left(\sigma_{1}-r_{j}-\gamma\right) S_{k_{j}-1}\left(r_{j}\right), \quad j=1,2,3, \\
t^{2}\left(\gamma^{2}-\left(\sigma_{1}+2\right) \gamma+\sigma_{2}+4\right) & =4+\sigma_{3}-2 \sigma_{2}-\sigma_{1}^{2},
\end{aligned}
$$

where $t:=t_{1}$ and $\gamma:=t^{2}+1-r / t$.

2.3. $\mathbf{S L}_{2}(\mathbb{R})$-representations. We now study $\mathrm{SL}_{2}(\mathbb{R})$-representations of the pretzel knot $P\left(a_{1}, a_{2}, a_{3}\right)$ by finding real solutions of the system of equations $(2.1)-(2.2)$.

2.3.1. Solving $r_{1}, r_{2}, r_{3}, \gamma$. We first consider real solutions of the system (2.1). Moreover we are interested in the case $r_{1}, r_{2}, r_{3} \in(2, \infty)$.

Let $p_{j}:=\frac{S_{k_{j}}\left(r_{j}\right)}{S_{k_{j}-1}\left(r_{j}\right)}$ for $j=1,2,3$. Then $p_{j}>1$ and $(2.1)$ becomes $\gamma=\frac{\sigma_{1}-r_{j}+\left(r_{j}+2\right) p_{j}}{p_{j}+1}$ for $j=1,2,3$. Hence the triple $\left(r_{1}, r_{2}, r_{3}\right) \in(2, \infty)^{3}$ satisfies

$$
\frac{r_{2}+r_{3}+\left(r_{1}+2\right) p_{1}}{p_{1}+1}=\frac{r_{3}+r_{1}+\left(r_{2}+2\right) p_{2}}{p_{2}+1}=\frac{r_{1}+r_{2}+\left(r_{3}+2\right) p_{3}}{p_{3}+1} .
$$

It is easy to check that this system is equivalent to

$$
\begin{aligned}
& \left(r_{1}-2\right)\left(p_{2}-p_{3}\right)=\left(r_{2}-r_{3}\right)\left(p_{2} p_{3}-1\right), \\
& \left(r_{2}-2\right)\left(p_{1}-p_{3}\right)=\left(r_{1}-r_{3}\right)\left(p_{1} p_{3}-1\right), \\
& \left(r_{3}-2\right)\left(p_{1}-p_{2}\right)=\left(r_{1}-r_{2}\right)\left(p_{1} p_{2}-1\right) .
\end{aligned}
$$

For example, the equation $\frac{r_{2}+r_{3}+\left(r_{1}+2\right) p_{1}}{p_{1}+1}=\frac{r_{3}+r_{1}+\left(r_{2}+2\right) p_{2}}{p_{2}+1}$ is equivalent to (2.5). Note that we actually just need two of the three equations (2.3)-(2.5).

Proposition 2.4. Suppose $k_{1}<k_{3}$. Then for every $r_{1} \in(2, \infty)$, there exist a unique $r_{3} \in\left(2, r_{1}\right)$ such that the triple $\left(r_{1}, r_{2}, r_{3}\right)$, where $r_{2}=2+\left(r_{1}-r_{3}\right) \frac{p_{1} p_{3}-1}{p_{1}-p_{3}}$, satisfies equations (2.3)-(2.5). Moreover $r_{3}$ is a continuous function in $r_{1} \in(2, \infty)$.

Proof. Fix $r_{1} \in(2, \infty)$. Consider $r_{3} \in\left(2, r_{1}\right)$. Since $1 \leq k_{1}<k_{3}$ and $2<r_{3}<r_{1}$, by Lemmas 2.2-2.3 we have $p_{1}=\frac{S_{k_{1}}\left(r_{1}\right)}{S_{k_{1}-1}\left(r_{1}\right)}>\frac{S_{k_{3}}\left(r_{3}\right)}{S_{k_{3}-1}\left(r_{3}\right)}=p_{3}>1$. From (2.4) we get

$$
r_{2}=2+\left(r_{1}-r_{3}\right) \frac{p_{3} p_{1}-1}{p_{1}-p_{3}}>2
$$


Since $r_{1}-r_{2}=r_{1}-2-\left(r_{1}-r_{3}\right) \frac{p_{1} p_{3}-1}{p_{1}-p_{3}}=r_{3}-2-\left(r_{1}-r_{3}\right)\left(\frac{p_{1} p_{3}-1}{p_{1}-p_{3}}-1\right)=r_{3}-2-$ $\left(r_{1}-r_{3}\right) \frac{\left(p_{1}+1\right)\left(p_{3}-1\right)}{p_{1}-p_{3}}$, equation $(2.5)$ then becomes

$$
\begin{aligned}
& \left(r_{3}-2\right)\left(p_{1}-p_{2}\right)=\left(r_{3}-2-\left(r_{1}-r_{3}\right) \frac{\left(p_{1}+1\right)\left(p_{3}-1\right)}{p_{1}-p_{3}}\right)\left(p_{1} p_{2}-1\right) \\
\Longleftrightarrow & \left(r_{1}-r_{3}\right) \frac{\left(p_{1}+1\right)\left(p_{3}-1\right)\left(p_{1} p_{2}-1\right)}{p_{1}-p_{3}}-\left(r_{3}-2\right)\left(p_{1}+1\right)\left(p_{2}-1\right)=0 \\
\Longleftrightarrow & \left(r_{1}-r_{3}\right) \frac{p_{1} p_{2}-1}{p_{2}-1}-\left(r_{3}-2\right) \frac{p_{1}-p_{3}}{p_{3}-1}=0 \\
\Longleftrightarrow & \left(r_{1}-r_{3}\right)\left(\frac{\left(p_{1}-1\right) p_{2}}{p_{2}-1}+1\right)-\left(r_{3}-2\right)\left(\frac{p_{1}-1}{p_{3}-1}-1\right)=0 \\
\Longleftrightarrow & \left(r_{1}-r_{3}\right) \frac{\left(p_{1}-1\right) p_{2}}{p_{2}-1}-\left(r_{3}-2\right) \frac{p_{1}-1}{p_{3}-1}+r_{1}-2=0 \\
\Longleftrightarrow & \frac{r_{3}-2}{p_{3}-1}-\left(r_{1}-r_{3}\right)\left(1+\frac{1}{p_{2}-1}\right)-\frac{r_{1}-2}{p_{1}-1}=0 .
\end{aligned}
$$

Consider the function $f:\left(2, r_{1}\right) \rightarrow \mathbb{R}$ defined by

$$
f\left(r_{3}\right)=\frac{r_{3}-2}{p_{3}-1}-\left(r_{1}-r_{3}\right)-\frac{r_{1}-r_{3}}{p_{2}-1}-\frac{r_{1}-2}{p_{1}-1} .
$$

We claim that $f$ is a strictly increasing function in $r_{3} \in\left(2, r_{1}\right)$. Indeed, we have

$$
\begin{aligned}
\frac{d f}{d r_{3}} & =\frac{d}{d r_{3}}\left(\frac{r_{3}-2}{p_{3}-1}\right)+1-\frac{d}{d r_{3}}\left(\frac{r_{1}-r_{3}}{p_{2}-1}\right) \\
& =\frac{d}{d r_{3}}\left(\frac{r_{3}-2}{p_{3}-1}\right)+1+\frac{\left(r_{1}-r_{3}\right) \frac{d p_{2}}{d r_{2}} \frac{d r_{2}}{d r_{3}}+\left(p_{2}-1\right)}{\left(p_{2}-1\right)^{2}},
\end{aligned}
$$

and

$$
\begin{aligned}
\frac{d r_{2}}{d r_{3}} & =\frac{d}{d r_{3}}\left(2+\left(r_{1}-r_{3}\right) \frac{p_{1} p_{3}-1}{p_{1}-p_{3}}\right) \\
& =\left(r_{1}-r_{3}\right) \frac{d}{d r_{3}}\left(\frac{p_{1} p_{3}-1}{p_{1}-p_{3}}\right)-\frac{p_{1} p_{3}-1}{p_{1}-p_{3}} \\
& =\frac{\left(r_{1}-r_{3}\right)\left(p_{1}^{2}-1\right)}{\left(p_{1}-p_{3}\right)^{2}} \frac{d p_{3}}{d r_{3}}-\frac{r_{2}-2}{r_{1}-r_{3}} .
\end{aligned}
$$

Hence, by noting that $\frac{\left(p_{2}-1\right)-\left(r_{2}-2\right) \frac{d p_{2}}{d r_{2}}}{\left(p_{2}-1\right)^{2}}=\frac{d}{d r_{2}}\left(\frac{r_{2}-2}{p_{2}-1}\right)$, we obtain

$$
\frac{d f}{d r_{3}}=\frac{d}{d r_{3}}\left(\frac{r_{3}-2}{p_{3}-1}\right)+1+\frac{\left(r_{1}-r_{3}\right)^{2}\left(p_{1}^{2}-1\right)}{\left(p_{1}-p_{3}\right)^{2}\left(p_{2}-1\right)^{2}} \frac{d p_{3}}{d r_{3}} \frac{d p_{2}}{d r_{2}}+\frac{d}{d r_{2}}\left(\frac{r_{2}-2}{p_{2}-1}\right)>1 .
$$

Here we used the facts that $\frac{d p_{2}}{d r_{2}}>0, \frac{d p_{3}}{d r_{3}}>0, \frac{d}{d r_{2}}\left(\frac{r_{2}-2}{p_{2}-1}\right)>0$ and $\frac{d}{d r_{3}}\left(\frac{r_{3}-2}{p_{3}-1}\right)>0$ in the last inequality. These facts follow from Lemma 2.3 . 
We have proved that $f\left(r_{3}\right)$ is a strictly increasing function in $r_{3} \in\left(2, r_{1}\right)$. Note that

$$
\begin{aligned}
\lim _{r_{3} \rightarrow 2^{+}} f\left(r_{3}\right) & =-\left(r_{1}-2\right)\left(\frac{p_{2}}{p_{2}-1}+\frac{1}{p_{1}-1}\right)<0, \\
\lim _{r_{3} \rightarrow r_{1}^{-}} f\left(r_{3}\right) & =\left(r_{1}-2\right)\left(\frac{1}{p_{3}-1}-\frac{1}{p_{1}-1}\right)>0 .
\end{aligned}
$$

Hence there exists a unique $r_{3} \in\left(2, r_{1}\right)$ such that $f\left(r_{3}\right)=0$. By the Implicit Function Theorem, $r_{3}$ is a continuous function in $r_{1} \in(2, \infty)$.

From now on, we consider the triple $\left(r_{1}, r_{2}, r_{3}\right) \in(2, \infty)^{3}$ in Proposition 2.4 when $k_{1}<k_{3}$. If $k_{1}=k_{3}$, by letting $r_{1}=r_{2}=r_{3}>2$ the equations (2.3)-(2.5) are satisfied.

Lemma 2.5. We have $r_{1}+r_{2}>r_{3}+2, r_{2}+r_{3}>r_{1}+2$ and $r_{3}+r_{1}>r_{2}+2$.

Proof. The case $k_{1}=k_{3}$ is obvious, so we only consider the case $k_{1}<k_{3}$. Note that if $x, y>1$ then $\left|\frac{x y-1}{x-y}\right|>1$. Since $r_{2}=2+\left(r_{1}-r_{3}\right) \frac{p_{1} p_{3}-1}{p_{1}-p_{3}}$ we have $r_{2}-2=\left|r_{1}-r_{3}\right|\left|\frac{p_{1} p_{3}-1}{p_{1}-p_{3}}\right|>$ $\left|r_{1}-r_{3}\right|$. This implies that $r_{2}+r_{1}>r_{3}+2$ and $r_{2}+r_{3}>r_{1}+2$.

Since $p_{1} \neq p_{3}$ we have either $p_{1} \neq p_{2}$ or $p_{3} \neq p_{2}$. If $p_{1} \neq p_{2}$ then $r_{3}-2=\mid r_{1}-$ $r_{2}|| \frac{p_{1} p_{2}-1}{p_{1}-p_{2}}|>| r_{1}-r_{2} \mid$, which implies that $r_{3}+r_{1}>r_{2}+2$. Similarly, if $p_{1} \neq p_{3}$ then $r_{1}-2=\left|r_{2}-r_{3}\right|\left|\frac{p_{2} p_{3}-1}{p_{2}-p_{3}}\right|>\left|r_{2}-r_{3}\right|$, which also implies that $r_{3}+r_{1}>r_{2}+2$.

Lemma 2.6. We have

$$
\begin{aligned}
& \lim _{r_{1} \rightarrow 2^{+}} \frac{r_{2}-2}{r_{1}-2}=\frac{1+k_{1}+k_{3}}{1+k_{2}+k_{3}}, \\
& \lim _{r_{1} \rightarrow 2^{+}} \frac{r_{3}-2}{r_{1}-2}=\frac{1+k_{1}+k_{2}}{1+k_{2}+k_{3}} .
\end{aligned}
$$

Proof. The case $k_{1}=k_{3}$ is obvious, so it suffices to consider the case $k_{1}<k_{3}$. Since $r_{3} \in\left(2, r_{1}\right)$ and $r_{1}+r_{3}-2>r_{2}>2$, we have $r_{3}, r_{2} \rightarrow 2^{+}$as $r_{1} \rightarrow 2^{+}$. This implies that $p_{j} \rightarrow \frac{S_{k_{j}}(2)}{S_{k_{j}-1}(2)}=\frac{k_{j}+1}{k_{j}}=1+\frac{1}{k_{j}}$ as $r_{1} \rightarrow 2^{+}$. Hence

$$
\lim _{r_{1} \rightarrow 2^{+}} \frac{r_{1}-r_{2}}{r_{3}-2}=\lim _{r_{1} \rightarrow 2^{+}} \frac{p_{1}-p_{2}}{p_{1} p_{2}-1}=\frac{k_{2}-k_{1}}{k_{1}+k_{2}+1} .
$$

Similarly, we have

$$
\lim _{r_{1} \rightarrow 2^{+}} \frac{r_{1}-r_{3}}{r_{2}-2}=\frac{k_{3}-k_{1}}{k_{1}+k_{3}+1} .
$$

Let $q_{2}=\lim _{r_{1} \rightarrow 2^{+}} \frac{r_{2}-2}{r_{1}-2}$ and $q_{3}=\lim _{r_{1} \rightarrow 2^{+}} \frac{r_{3}-2}{r_{1}-2}$. From (2.6) and (2.7) we have

$$
\frac{1-q_{2}}{q_{3}}=\frac{k_{2}-k_{1}}{k_{1}+k_{2}+1} \quad \text { and } \quad \frac{1-q_{3}}{q_{2}}=\frac{k_{3}-k_{1}}{k_{1}+k_{3}+1} .
$$

Hence $q_{2}=\frac{1+k_{1}+k_{3}}{1+k_{2}+k_{3}}$ and $q_{3}=\frac{1+k_{1}+k_{2}}{1+k_{2}+k_{3}}$ as claimed.

Let $\gamma:=\frac{r_{2}+r_{3}+\left(r_{1}+2\right) p_{1}}{p_{1}+1}$ and $r:=x^{3}+x-x \gamma$. Then the system (2.1) is satisfied.

Lemma 2.7. We have $\sigma_{1}+2-2 \gamma>0$. 
Proof. This is because

$$
\sigma_{1}+2-2 \gamma=r_{2}+r_{2}+r_{3}+2-\frac{r_{2}+r_{3}+\left(r_{1}+2\right) p_{1}}{p_{1}+1}=\frac{\left(r_{2}+r_{3}-r_{1}-2\right)\left(p_{1}-1\right)}{p_{1}+1} .
$$

and $r_{2}+r_{3}-r_{1}-2>0$ (by Lemma 2.5).

2.3.2. Trace of meridian. We now study the equation (2.2). Recall that $\sigma_{1}=r_{1}+r_{2}+r_{3}$, $\sigma_{2}=r_{1} r_{2}+r_{2} r_{3}+r_{3} r_{1}$ and $\sigma_{3}=r_{1} r_{2} r_{3}$.

Let $\delta:=r_{1} r_{2} r_{3}+4-r_{1}^{2}-r_{2}^{2}-r_{3}^{2}=4+\sigma_{3}-2 \sigma_{2}-\sigma_{1}^{2}$ be the RHS of $(2.2)$.

Lemma 2.8. We have $\delta>\left(r_{1}-2\right)\left(r_{2}-2\right)\left(r_{3}-2\right)>0$.

Proof. Let $a_{j}=r_{j}-2>0$. By Lemma 2.5 we have $a_{1}+a_{2}>a_{3}, a_{2}+a_{3}>a_{1}$ and $a_{3}+a_{1}>a_{2}$. Hence

$$
\begin{aligned}
\delta & =\left(a_{1}+2\right)\left(a_{2}+2\right)\left(a_{3}+2\right)+4-\left(a_{1}+2\right)^{2}-\left(a_{2}+2\right)^{2}-\left(a_{3}+2\right)^{2} \\
& =a_{1} a_{2} a_{3}+2 a_{1} a_{2}+2 a_{2} a_{3}+2 a_{3} a_{1}-a_{1}^{2}-a_{2}^{2}-a_{3}^{2} \\
& =a_{1} a_{2} a_{3}+\left(a_{1}+a_{2}-a_{3}\right) a_{3}+\left(a_{2}+a_{3}-a_{1}\right) a_{1}+\left(a_{3}+a_{1}-a_{2}\right) a_{2} \\
& >a_{1} a_{2} a_{3}>0 .
\end{aligned}
$$

The lemma follows.

For the LHS of (2.2) we have the following.

Lemma 2.9. We have

$$
\gamma^{2}-\left(\sigma_{1}+2\right) \gamma+\sigma_{2}+4=\left(r_{2}-2\right)\left(r_{3}-2\right)-\frac{\left(r_{2}+r_{3}-r_{1}-2\right)^{2} p_{1}}{\left(p_{1}+1\right)^{2}} .
$$

Proof. We have $\gamma-\left(\sigma_{1}+2\right)=\frac{r_{2}+r_{3}+\left(r_{1}+2\right) p_{1}}{p_{1}+1}-\left(r_{2}+r_{3}+r_{1}+2\right)=-\frac{r_{1}+2+\left(r_{2}+r_{3}\right) p_{1}}{p_{1}+1}$. This implies that

$$
\begin{aligned}
\gamma^{2}-\left(\sigma_{1}+2\right) \gamma & =-\frac{r_{1}+2+\left(r_{2}+r_{3}\right) p_{1}}{p_{1}+1} \cdot \frac{r_{2}+r_{3}+\left(r_{1}+2\right) p_{1}}{p_{1}+1} \\
& =-\left(\left(r_{1}+2\right)\left(r_{2}+r_{3}\right)+\frac{\left(r_{2}+r_{3}-r_{1}-2\right)^{2} p_{1}}{\left(p_{1}+1\right)^{2}}\right) .
\end{aligned}
$$

The lemma then follows, since $\sigma_{2}+4-\left(r_{1}+2\right)\left(r_{2}+r_{3}\right)=\left(r_{2}-2\right)\left(r_{3}-2\right)$.

Since $S_{k_{1}}^{2}\left(r_{1}\right)+S_{k_{1}-1}^{2}\left(r_{1}\right)=r_{1} S_{k_{1}}\left(r_{1}\right) S_{k_{1}-1}\left(r_{1}\right)+1$ (by Lemma 2.1) we have

$$
\frac{\left(p_{1}+1\right)^{2}}{p_{1}}=\frac{S_{k_{1}}\left(r_{1}\right)}{S_{k_{1}-1}\left(r_{1}\right)}+\frac{S_{k_{1}-1}\left(r_{1}\right)}{S_{k_{1}}\left(r_{1}\right)}+2=\frac{1}{S_{k_{1}}\left(r_{1}\right) S_{k_{1}-1}\left(r_{1}\right)}+r_{1}+2>r_{1}+2 .
$$

This implies that $\frac{p_{1}}{\left(p_{1}+1\right)^{2}}<\frac{1}{r_{1}+2}$. Hence, by Lemma 2.9 we obtain

$$
\gamma^{2}-\left(\sigma_{1}+2\right) \gamma+\sigma_{2}+4>\left(r_{2}-2\right)\left(r_{3}-2\right)-\frac{\left(r_{2}+r_{3}-r_{1}-2\right)^{2}}{r_{1}+2}=\frac{\delta}{r_{1}+2}>0 .
$$

Let $T=\frac{\delta}{\gamma^{2}-\left(\sigma_{1}+2\right) \gamma+\sigma_{2}+4}>0$ and $t= \pm \sqrt{T}$. Then the equation (2.2) is satisfied.

Lemma 2.10. We have $r_{1}-2<T<r_{1}+2$.

Proof. Note that $T<r_{1}+2$ by (2.8). Since $0<\gamma^{2}-\left(\sigma_{1}+2\right) \gamma+\sigma_{2}+4<\left(r_{2}-2\right)\left(r_{3}-2\right)$ (by Lemma 2.9) and $\delta>\left(r_{1}-2\right)\left(r_{2}-2\right)\left(r_{3}-2\right)>0$ (by Lemma 2.8) we have $T>r_{1}-2$. 
Proposition 2.11. We have $\lim _{r_{1} \rightarrow \infty} T=\infty$ and

$$
\lim _{r_{1} \rightarrow 2^{+}} T=4-\frac{1}{1+k_{1}+k_{2}+k_{3}+k_{1} k_{2}+k_{2} k_{3}+k_{3} k_{1}} .
$$

Proof. Since $T>r_{1}-2$ (by Lemma 2.10) we have $\lim _{r_{1} \rightarrow \infty} T=\infty$.

Let $a_{j}=r_{j}-2>0$ for $j=1,2,3$. By Lemma 2.6 we have $\lim _{a_{1} \rightarrow 0^{+}} \frac{a_{2}}{a_{1}}=q_{2}$ and $\lim _{a_{1} \rightarrow 0^{+}} \frac{a_{3}}{a_{1}}=q_{3}$, where $q_{2}=\frac{1+k_{1}+k_{3}}{1+k_{2}+k_{3}}$ and $q_{3}=\frac{1+k_{1}+k_{2}}{1+k_{2}+k_{3}}$.

From the proof of Lemma 2.8 we have $\delta=a_{1} a_{2} a_{3}+4 a_{2} a_{3}-\left(a_{2}+a_{3}-a_{1}\right)^{2}$. By Lemma 2.9 we have $\gamma^{2}-\left(\sigma_{1}+2\right) \gamma+\sigma_{2}+4=a_{2} a_{3}-\left(a_{2}+a_{3}-a_{1}\right)^{2} /\left(p_{1}+p_{1}^{-1}+2\right)$. Hence

$$
\begin{aligned}
\lim _{r_{1} \rightarrow 2^{+}} T & =\lim _{a_{1} \rightarrow 0^{+}} \frac{a_{1} a_{2} a_{3}+4 a_{2} a_{3}-\left(a_{2}+a_{3}-a_{1}\right)^{2}}{a_{2} a_{3}-\left(a_{2}+a_{3}-a_{1}\right)^{2} /\left(p_{1}+p_{1}^{-1}+2\right)} \\
& =\frac{4 q_{2} q_{3}-\left(q_{2}+q_{3}-1\right)^{2}}{q_{2} q_{3}-\left(q_{2}+q_{3}-1\right)^{2} /\left(\frac{k_{1}+1}{k_{1}}+\frac{k_{1}}{k_{1}+1}+2\right)} .
\end{aligned}
$$

Since $q_{2}+q_{3}=1+\frac{1+2 k_{1}}{1+k_{2}+k_{3}}$ and $q_{2} q_{3}=\frac{\left(1+k_{1}+k_{3}\right)\left(1+k_{1}+k_{2}\right)}{\left(1+k_{2}+k_{3}\right)^{2}}$ we have

$$
\begin{aligned}
4 q_{2} q_{3}-\left(q_{2}+q_{3}-1\right)^{2} & =\frac{4\left(1+k_{1}+k_{3}\right)\left(1+k_{1}+k_{2}\right)}{\left(1+k_{2}+k_{3}\right)^{2}}-\frac{\left(2 k_{1}+1\right)^{2}}{\left(1+k_{2}+k_{3}\right)^{2}} \\
& =\frac{3+4 k_{1}+4 k_{2}+4 k_{3}+4 k_{1} k_{2}+4 k_{2} k_{3}+4 k_{3} k_{1}}{\left(1+k_{2}+k_{3}\right)^{2}},
\end{aligned}
$$

and

$$
\begin{aligned}
q_{2} q_{3}-\frac{\left(q_{2}+q_{3}-1\right)^{2}}{\frac{k_{1}+1}{k_{1}}+\frac{k_{1}}{k_{1}+1}+2} & =\frac{\left(1+k_{1}+k_{3}\right)\left(1+k_{1}+k_{2}\right)}{\left(1+k_{2}+k_{3}\right)^{2}}-\frac{k_{1}\left(k_{1}+1\right)}{\left(1+k_{2}+k_{3}\right)^{2}} \\
& =\frac{1+k_{1}+k_{2}+k_{3}+k_{1} k_{2}+k_{2} k_{3}+k_{3} k_{1}}{\left(1+k_{2}+k_{3}\right)^{2}} .
\end{aligned}
$$

Hence $\lim _{r_{1} \rightarrow 2^{+}} T=4-1 /\left(1+k_{1}+k_{2}+k_{3}+k_{1} k_{2}+k_{2} k_{3}+k_{3} k_{1}\right)$.

Since $a_{j}=2 k_{j}+1$, we also have $\lim _{r_{1} \rightarrow 2^{+}} T=4-4 /\left(1+a_{1} a_{2}+a_{2} a_{3}+a_{3} a_{1}\right)$. If we let

$$
\theta_{0}=\frac{1}{2} \arccos \left(1-2 /\left(1+a_{1} a_{2}+a_{2} a_{3}+a_{3} a_{1}\right)\right) \in\left(0, \frac{\pi}{2}\right)
$$

then $\lim _{r_{1} \rightarrow 2^{+}} T=2+2 \cos 2 \theta=4 \cos ^{2} \theta_{0}$.

\section{LEFT-ORDERABILITY}

In this section we will use the continuous family of irreducible $\mathrm{SL}_{2}(\mathbb{R})$-representations constructed in the previous section to study the left-orderabilty of the fundamental groups of the 3-manifolds obtained by Dehn surgeries on $P\left(a_{1}, a_{2}, a_{3}\right)$ and to study the leftorderabilty of the fundamental groups of the cyclic branched covers of $P\left(a_{1}, a_{2}, a_{3}\right)$, where $a_{1}, a_{2}, a_{3}$ are positive odd integers. In particular, we will use elliptic $\mathrm{SL}_{2}(\mathbb{R})$ representations to give a proof of Theorem 1 .

Recall that we have considered the triple $\left(r_{1}, r_{2}, r_{3}\right) \in(2, \infty)^{3}$ in Proposition 2.4 when $k_{1}<k_{3}$. If $k_{1}=k_{3}$ we let $r_{1}=r_{2}=r_{3}>2$. Note that $r_{3}, r_{2}$ are continuous functions in $r_{1} \in(2, \infty)$. In both cases, for each $r_{1} \in(2, \infty)$ we let $r=x^{3}+x-x \gamma, t= \pm \sqrt{T}$ where

$$
\gamma=\frac{r_{2}+r_{3}+\left(r_{1}+2\right) p_{1}}{p_{1}+1}, \quad T=\frac{\delta}{\gamma^{2}-\left(\sigma_{1}+2\right) \gamma+\sigma_{2}+4}>0 .
$$


Note that $\sigma_{1}+2-2 \gamma>0$ by Lemma 2.7. Then the system (2.1) $-(2.2)$ is satisfied, and hence there exists an irreducible representation $\rho^{ \pm}=\rho_{r_{1}}^{ \pm}: G(K) \rightarrow \mathrm{SL}_{2}(\mathbb{C})$ with

$$
\left(\operatorname{tr} \rho^{ \pm}\left(x_{1}\right), \operatorname{tr} \rho^{ \pm}\left(x_{2} x_{3}^{-1}\right), \operatorname{tr} \rho^{ \pm}\left(x_{3} x_{1}^{-1}\right), \operatorname{tr} \rho^{ \pm}\left(x_{1} x_{2}^{-1}\right), \operatorname{tr} \rho^{ \pm}\left(x_{1} x_{2} x_{3}\right)\right)=\left( \pm \sqrt{T}, r_{1}, r_{2}, r_{3}, r\right) .
$$

Since $\rho^{ \pm}$has real traces, it is conjugate to either an $\mathrm{SL}_{2}(\mathbb{R})$ representation or an $\mathrm{SU}(2)$ representation, see e.g. [HP, Lemma 10.1]. If the latter occurs, then $\left|\operatorname{tr} \rho^{ \pm}(g)\right|<2$ for all $g \in G(K)$. Since $\operatorname{tr} \rho_{r_{1}}^{ \pm}\left(x_{2} x_{3}^{-1}\right)=r_{1}>2, \rho^{ \pm}$is conjugate to an $\mathrm{SL}_{2}(\mathbb{R})$ representation.

3.1. Elliptic $\mathbf{S L}_{2}(\mathbb{R})$-representations. Let $X$ be the complement of an open tubular neighborhood of $K:=P\left(a_{1}, a_{2}, a_{3}\right)$ in $S^{3}$, and $X_{m / l}$ the 3 -manifold obtained from $S^{3}$ by $\frac{m}{l}$-surgery along $K$.

An element of $\mathrm{SL}_{2}(\mathbb{R})$ is called elliptic if its trace is a real number in $(-2,2)$. A representation $\rho: \mathbb{Z}^{2} \rightarrow \mathrm{SL}_{2}(\mathbb{R})$ is called elliptic if the image group $\rho\left(\mathbb{Z}^{2}\right)$ contains an elliptic element of $\mathrm{SL}_{2}(\mathbb{R})$. In which case, since $\mathbb{Z}^{2}$ is an abelian group every non-trivial element of $\rho\left(\mathbb{Z}^{2}\right)$ must also be elliptic.

Proposition 3.1. For each rational number $\frac{m}{l} \in(-\infty, 0) \cup(0,1)$ there exists a representation $\rho: \pi_{1}\left(X_{m / l}\right) \rightarrow \mathrm{SL}_{2}(\mathbb{R})$ such that $\left.\rho\right|_{\pi_{1}(\partial X)}: \pi_{1}(\partial X) \cong \mathbb{Z}^{2} \rightarrow \mathrm{SL}_{2}(\mathbb{R})$ is an elliptic representation.

Proof. Since $\lim _{r_{1} \rightarrow 2^{+}} T=4 \cos ^{2} \theta_{0}<4$ and $\lim _{r_{1} \rightarrow \infty} T=\infty$ (by Proposition 2.11), there exists $r_{1}^{*}>2$ such that $T\left(r_{1}^{*}\right)=4$ and for all $r_{1} \in\left(2, r_{1}^{*}\right)$ we have $0<T\left(r_{1}\right)<4$.

For each $r_{1} \in\left(2, r_{1}^{*}\right)$ we let $\theta=\arccos (\sqrt{T} / 2) \in(0, \pi / 2)$. Note that $\lim _{r_{1} \rightarrow 2^{+}} \theta=\theta_{0}$ and $\lim _{r_{1} \rightarrow r_{1}^{*}} \theta=0$. Then $\operatorname{tr} \rho^{ \pm}\left(x_{1}\right)=\sqrt{T}= \pm 2 \cos \theta= \pm\left(e^{i \theta}+e^{-i \theta}\right)$.

Let $\lambda$ be the canonical longitude corresponding to the meridian $\mu:=x_{1}$. Up to conjugation we assume that

$$
\rho^{+}(\mu)=\left[\begin{array}{cc}
M & * \\
0 & M^{-1}
\end{array}\right] \text { and } \rho^{+}(\lambda)=\left[\begin{array}{cc}
L & * \\
0 & L^{-1}
\end{array}\right]
$$

where $M:=e^{i \theta}$. By [Ha, Proposition 4.1] $L$ satisfies the following equation

$$
(1+L)\left(M+M^{-1}\right)\left(\sigma_{1}+2-2 \gamma\right)=(1-L)\left(M-M^{-1}\right)\left(\sigma_{1}+2-2 t^{2}\right) .
$$

This can be rewritten as $L\left(\alpha M-\beta M^{-1}\right)+\alpha M^{-1}-\beta M=0$, where

$$
\alpha:=\sigma_{1}+2-\gamma-t^{2}, \quad \beta:=\gamma-t^{2} .
$$

Note that $\alpha, \beta \in \mathbb{R}$. By Lemmas 2.5 and 2.10 we have

$$
\beta=\frac{\left(r_{1}+2\right) w_{1}+\left(r_{2}+r_{3}\right) v_{1}}{v_{1}+w_{1}}-t^{2}=\frac{\left(r_{2}+r_{3}-r_{1}-2\right) v_{1}}{v_{1}+w_{1}}+r_{1}+2-t^{2}>0 .
$$

Moreover, we have $\alpha-\beta=\sigma_{1}+2-2 \gamma>0$ by Lemma 2.7. Hence $\alpha>\beta>0$.

We have $\left|\alpha M-\beta M^{-1}\right|^{2}=\alpha^{2}+\beta^{2}-2 \alpha \beta \cos \theta=(\alpha-\beta)^{2}+2 \alpha \beta(1-\cos \theta)>0$, which implies that $\alpha M-\beta M^{-1} \neq 0$. Hence $L=-\left(\alpha M^{-1}-\beta M\right) /\left(\alpha M-\beta M^{-1}\right)$.

Since $\alpha M^{-1}-\beta M$ and $\alpha M-\beta M^{-1}$ are complex conjugates, $L$ is a unit complex number. Moreover, by a direct calculation we have

$$
\begin{aligned}
& \operatorname{Re}(L)=\left(2 \alpha \beta-\left(\alpha^{2}+\beta^{2}\right) \cos 2 \theta\right) /\left(\alpha^{2}+\beta^{2}-2 \alpha \beta \cos \theta\right), \\
& \operatorname{Im}(L)=\left(\alpha^{2}-\beta^{2}\right) \sin 2 \theta /\left(\alpha^{2}+\beta^{2}-2 \alpha \beta \cos \theta\right) .
\end{aligned}
$$

Note that $\operatorname{Im}(L)>0$ and so $L=e^{i \varphi}$, where

$$
\varphi:=\arccos \left(\left(2 \alpha \beta-\left(\alpha^{2}+\beta^{2}\right) \cos 2 \theta\right) /\left(\alpha^{2}+\beta^{2}-2 \alpha \beta \cos \theta\right)\right) \in(0, \pi) .
$$


As $r_{1} \rightarrow 2^{+}$we have $r_{3}, r_{2} \rightarrow 2^{+}$and $\theta \rightarrow \theta_{0}$. Note that $\gamma=\frac{r_{2}+r_{3}+\left(r_{1}+2\right) p_{1}}{p_{1}+1} \rightarrow 4$ and so $\alpha, \beta \rightarrow 4-4 \cos ^{2} \theta_{0}$. Hence $L=-\left(\alpha M^{-1}-\beta M\right) /\left(\alpha M-\beta M^{-1}\right) \rightarrow 1$ and $\varphi \rightarrow 0$.

As $r_{1} \rightarrow\left(r_{1}^{*}\right)^{-}$we have $T \rightarrow 4$ and $\theta \rightarrow 0^{+}$. Note that $\alpha \rightarrow \alpha\left(r_{1}^{*}\right)$ and $\beta \rightarrow \beta\left(r_{1}^{*}\right)$, where $\alpha\left(r_{1}^{*}\right)>\beta\left(r_{1}^{*}\right)>0$. Hence $L=-\left(\alpha M^{-1}-\beta M\right) /\left(\alpha M-\beta M^{-1}\right) \rightarrow-1$ and $\varphi \rightarrow \pi$.

Since $\lim _{r_{1} \rightarrow 2^{+}}\left(-\frac{\varphi}{\theta}\right)=0$ and $\lim _{r_{1} \rightarrow\left(r_{1}^{*}\right)^{-}}\left(-\frac{\varphi}{\theta}\right)=-\infty$, the image of the continuous function $-\frac{\varphi}{\theta}:\left(2, r_{1}^{*}\right) \rightarrow \mathbb{R}$ contains the interval $(-\infty, 0)$.

We now finish the proof of Proposition 3.1. First, suppose $\frac{m}{l} \in(-\infty, 0) \cap \mathbb{Q}$. By the above argument, $\frac{m}{l}=-\frac{\varphi\left(r_{1}\right)}{\theta\left(r_{1}\right)}$ for some $r_{1} \in\left(2, r_{1}^{*}\right)$. Since $M^{m} L^{l}=e^{i(m \theta+l \varphi)}=1$, we have $\rho^{+}\left(\mu^{m} \lambda^{l}\right)=I$. Let $\rho: G(K) \rightarrow \mathrm{SL}_{2}(\mathbb{R})$ be an $\mathrm{SL}_{2}(\mathbb{R})$ representation conjugate to $\rho^{+}$. Then we also have $\rho\left(\mu^{m} \lambda^{l}\right)=I$. This means that $\rho$ extends to a representation $\rho: \pi_{1}\left(X_{m / l}\right) \rightarrow \mathrm{SL}_{2}(\mathbb{R})$. Note that $\left.\rho\right|_{\pi_{1}(\partial X)}$ is an elliptic representation.

Finally, suppose $\frac{m}{l} \in(0,1) \cap \mathbb{Q}$. Consider $\rho^{-}=\rho^{-}\left(r_{1}\right)$ of the form

$$
\rho^{-}(\mu)=\left[\begin{array}{cc}
M^{\prime} & * \\
0 & \left(M^{\prime}\right)^{-1}
\end{array}\right] \text { and } \rho^{-}(\lambda)=\left[\begin{array}{cc}
L^{\prime} & * \\
0 & \left(L^{\prime}\right)^{-1}
\end{array}\right]
$$

where $M^{\prime}=-M=e^{i(\theta-\pi)}$. As above we have $L^{\prime}=-\frac{\alpha\left(M^{\prime}\right)^{-1}-\beta M^{\prime}}{\alpha M^{\prime}-\beta\left(M^{\prime}\right)^{-1}}=L$ and so $L^{\prime}=e^{i \varphi}$.

Since $\lim _{r_{1} \rightarrow 2^{+}}\left(-\frac{\varphi}{\theta-\pi}\right)=0$ and $\lim _{r_{1} \rightarrow\left(r_{1}^{*}\right)^{-}}\left(-\frac{\varphi}{\theta-\pi}\right)=1$, the image of the continuous function $-\frac{\varphi}{\pi-\theta}:\left(2, r_{1}^{*}\right) \rightarrow \mathbb{R}$ contains the interval $(0,1)$. This implies that $\frac{m}{l}=-\frac{\varphi\left(r_{1}\right)}{\theta\left(r_{1}\right)-\pi}$ for some $r_{1} \in\left(2, r_{1}^{*}\right)$. Since $\left(M^{\prime}\right)^{m}\left(L^{\prime}\right)^{l}=e^{i(m(\theta-\pi)+l \varphi)}=1$, we have $\rho^{-}\left(\mu^{m} \lambda^{l}\right)=I$.

Let $\rho^{\prime}: G(K) \rightarrow \mathrm{SL}_{2}(\mathbb{R})$ be an $\mathrm{SL}_{2}(\mathbb{R})$-representation conjugate to $\rho^{-}$. Then we also have $\rho^{\prime}\left(\mu^{m} \lambda^{l}\right)=I$. Hence $\rho^{\prime}$ extends to a representation $\rho^{\prime}: \pi_{1}\left(X_{m / n}\right) \rightarrow \mathrm{SL}_{2}(\mathbb{R})$. Note that $\left.\rho^{\prime}\right|_{\pi_{1}(\partial X)}$ is an elliptic representation.

3.2. Proof of Theorem 1. (i) Suppose $\frac{m}{l} \in(-\infty, 1) \cap \mathbb{Q}$. If $\frac{m}{l} \neq 0$, by Proposition 3.1 , there exists a representation $\rho: \pi_{1}\left(X_{m / l}\right) \rightarrow \mathrm{SL}_{2}(\mathbb{R})$ such that $\left.\rho\right|_{\pi_{1}(\partial X)}$ is an elliptic representation. This representation lifts to a representation $\tilde{\rho}: \pi_{1}\left(X_{m / l}\right) \rightarrow \widehat{\mathrm{SL}_{2}(\mathbb{R})}$, where $\widehat{\mathrm{SL}_{2}(\mathbb{R})}$ is the universal covering group of $\mathrm{SL}_{2}(\mathbb{R})$. See e.g. [CD, Section 3.5] and [Va, Section 2.2]. Note that $X_{m / l}$ is an irreducible 3-manifold (by [HTh]) and $\widehat{\mathrm{SL}_{2}(\mathbb{R})}$ is a left orderable group (by $[\mathrm{Be}]$ ). Hence, by $[\mathrm{BRW}], \pi_{1}\left(X_{m / l}\right)$ is a left orderable group. Finally, 0-surgery along a knot always produces a prime manifold whose first Betti number is 1, and by [BRW] such manifold has left orderable fundamental group.

(ii) Suppose $n>2 \pi / \arccos \left(1-2 /\left(1+a_{1} a_{2}+a_{2} a_{3}+a_{3} a_{1}\right)\right)=\pi / \theta_{0}$. Since $4 \cos ^{2} \frac{\pi}{n} \in$ $\left(4 \cos ^{2} \theta_{0}, 4\right)$, we have $4 \cos ^{2} \frac{\pi}{n}=T\left(r_{1}\right)$ for some $r_{1} \in\left(2, r_{1}^{*}\right)$. Consider $\rho^{+}:=\rho_{r_{1}}^{+}$. Then $\operatorname{tr} \rho^{+}(\mu)=\sqrt{T}=2 \cos \frac{\pi}{n}$. This implies that $e^{\frac{ \pm i \pi}{n}}$ are eigenvalues of $\rho^{+}(\mu)$. Hence $\rho^{+}(\mu)$ is conjugate to the diagonal matrix $D:=\operatorname{diag}\left(e^{\frac{i \pi}{n}}, e^{-\frac{i \pi}{n}}\right)$.

Let $\rho: G(K) \rightarrow \mathrm{SL}_{2}(\mathbb{R})$ be an irreducible $\mathrm{SL}_{2}(\mathbb{R})$-representation conjugate to $\rho^{+}$. Then $\rho(\mu)$ is conjugate to the diagonal matrix $D$. Since $D^{n}=-I$, we have $\rho\left(\mu^{n}\right)=-I$. By applying [BGW, Theorem 6] and [Hu1, Theorem 3.1] we conclude that the fundamendal group of the $n^{\text {th }}$-cyclic branched cover of $P\left(a_{1}, a_{2}, a_{3}\right)$ is left-orderable. 


\section{ACKNOWLEDGEMENTS}

This paper is essentially the Ph.D. thesis of the first author written under the supervision of the second author. The second author is partially supported by a grant from the Simons Foundation (\#354595).

\section{REFERENCES}

[BBG] M. Boileau, S. Boyer and C. Gordon, Branched covers of quasi-positive links and L-spaces, J. Topol. 12 (2019), no. 2, 536-576.

[Be] G. Bergman, Right orderable groups that are not locally indicable, Pacific J. Math. 147 (1991), no. 2, 243-248.

[BGW] S. Boyer, C. Gordon and L. Watson, On L-spaces and left-orderable fundamental groups, Math. Ann. 356 (2013), no. 4, 1213-1245.

[BRW] S. Boyer, D. Rolfsen and B. Wiest, Orderable 3-manifold groups, Ann. Inst. Fourier (Grenoble) 55 (2005), no. 1, 243-288.

[CD] M. Culler and N. Dunfield, Orderability and Dehn filling, Geom. Topol. 22 (2018), no. 3, 14051457.

[DPT] M. Dabkowski, J. Przytycki and A. Togha, Non-left-orderable 3-manifold groups, Canad. Math. Bull., 48 (2005), 32-40.

[Ga1] X. Gao, Orderability of homology spheres obtained by Dehn filling, arXiv:1810.11202.

[Ga2] X. Gao, Slope of orderable Dehn filling of two-bridge knots, arXiv:1912.07468.

[Go] C. Gordon, Riley's conjecture on $\mathrm{SL}(2, \mathbb{R})$ representations of 2-bridge knots, J. Knot Theory Ramifications, 26 (2017), 1740003.

[Ha] H. Chen, Character varieties of odd classical pretzel knots, Internat. J. Math. 29 (2018), no. 9, 1850060, 15 pp.

[HP] M. Heusener and J. Porti, Deformations of reducible representations of 3-manifold groups into $\mathrm{PSL}_{2} \mathbb{C}$ ), Algebr. Geom. Topol. 5 (2005), 965-997.

[HTh] A. Hatcher and W. Thurston, Incompressible surfaces in 2-bridge knot complements, Invent. Math. 79 (1985), no. 2, 225-246.

[HT1] R. Hakamata and M. Teragaito, Left-orderable fundamental group and Dehn surgery on the knot $5_{2}$, Canad. Math. Bull. 57 (2014), no. 2, 310-317.

[HT2] R. Hakamata and M. Teragaito, Left-orderable fundamental groups and Dehn surgery on genus one 2-bridge knots, Algebr. Geom. Topol. 14 (2014), no. 4, 2125-2148.

[Hu1] Y. Hu, The left-orderability and the cyclic branched coverings, Algebr. Geom. Topol. 15 (2015), 399-413.

[Hu2] Y. Hu, Euler class of taut foliations and Dehn filling, arXiv:1912.01645.

[KTT] V. Khoi, M. Teragaito and A. Tran, Left orderable surgeries of double twist knots II, Canad. Math. Bull., published online (August 2020), DOI: https://doi.org/10.4153/S0008439520000703. arXiv:2003.00623.

[OS] P. Ozsvath and Z. Szabo, On knot Floer homology and lens space surgeries, Topology 44 (2005), no. $6,1281-1300$.

[Ri] R. Riley, Nonabelian representations of 2-bridge knot groups, Quart. J. Math. Oxford Ser. (2) 35 (1984), no. 138, 191-208.

[Tr1] A. Tran, On left-orderable fundamental groups and Dehn surgeries on knots, J. Math. Soc. Japan 67 (2015), no. 1, 319-338.

[Tr2] A. Tran, On left-orderability and cyclic branched coverings, J. Math. Soc. Japan 67 (2015), no. $3,1169-1178$.

[Tr3] A. Tran, Nonabelian representations and signatures of double twist knots, J. Knot Theory Ramifications 25 (2016), 1640013, 9 pp.

[Tr4] A. Tran, Left orderable surgeries of double twist knots, J. Math. Soc. Japan, Advance publication (2020), 13 pages, DOI: 10.2969/jmsj/84058405. arXiv:1911.03798.

[Tu] H. Turner, Left-oderability, branched covers and double twist knots, arXiv:2002.10611.

[Va] K. Varvarezos, Representations of the $(-2,3,7)$-pretzel knot and orderability of Dehn surgeries, arXiv:1911.11745. 
[Zu] J. Zung, Taut foliations, left-orders, and pseudo-Anosov mapping tori, arXiv:2006.07706.

Department of Mathematical Sciences, The University of Texas at Dallas, RichardSON, TX 75080, USA

Email address: arafat@utdallas.edu

Department of Mathematical Sciences, The University of Texas at Dallas, RichardSON, TX 75080, USA

Email address: att140830@utdallas.edu 